\title{
Salmonella enterica: una revisión de la trilogía agente, hospedero y ambiente, y su trascendencia en Chile
}

\author{
Marlen Barreto, Mario Castillo-Ruiz y Patricio Retamal
}

\section{Salmonella enterica: a review or the trilogy agent, host and environment and its importance in Chile}

Salmonella enterica is a major foodborne pathogen worldwide, being the main cause of outbreaks by food consumption in Chile. Despite all efforts deployed for control and prevention, the high incidence in people still persists, with several factors that could be influencing the epidemiological behavior of this infection. The objective of this review is to identify these factors belonging to the biological agent, the human host and the environment, which probably have a greater importance in Chile. Thus, priority areas for research of $S$. enterica are inferred, which hopefully will help to understand its spread in nature and its success as a wide host range pathogen. In the future, increased understanding of these determinants will facilitate the implementation of biosecurity and surveillance strategies for the prevention of disease in people and animals.

Key words: Salmonella, zoonosis, foodborne diseases, predisposing factors, Chile.

Palabras clave: Salmonella, zoonosis, enfermedad transmitida por alimentos, factores determinantes, Chile.
Universidad Autónoma de Chile.

Instituto de Investigación Biomédica (MB).

Universidad Andrés Bello, Facultad de Medicina, Escuela de Química y Farmacia (MC-R). Universidad de Chile. Facultad de Ciencias Veterinarias y Pecuarias (PR).

Los autores declaran que no existen conflictos de interés asociados a esta publicación.

Recibido: 11 de diciembre de 2015 Aceptado: 1 de septiembre de 2016

Correspondencia a:

Patricio Retamal Merino pretamal@uchile.cl

\section{Introducción}

L as enfermedades emergentes (EE) se definen como aquellas patologías que han aparecido o se han descrito por primera vez en los últimos 30 años, en contraste con las enfermedades re-emergentes que ya se conocían con anterioridad, pero que últimamente, y como consecuencia de diversos factores, han incrementado significativamente su incidencia ${ }^{1-3}$. La mayoría de las EE son provocadas por agentes biológicos zoonóticos, es decir, que se transmiten naturalmente entre las personas y los animales, ya sea en forma directa mediante mordeduras o manipulación de éstos o bien, de forma indirecta por la participación de vectores, o por el consumo de alimentos contaminados ${ }^{4}$. Entre los patógenos transmitidos por los alimentos, Salmonella enterica es uno de los agentes más relevantes debido a su impacto en la salud pública y la sanidad animal, tanto en países desarrollados como en vías de desarrollo ${ }^{5}$. En este sentido, Salmonella es el agente etiológico bacteriano más frecuente en los brotes transmitidos por alimentos; así es en Chile ${ }^{6}$, como en la Unión Europea y E.U.A. ${ }^{7,8}$.

El género Salmonella pertenece a la familia Enterobacteriaceae y está constituido por bacterias gramnegativas, intracelulares facultativas, que se han agrupado en las especies S. enterica y $S$. bongori. Sin embargo, el potencial patogénico está representado por $S$. enterica y por sus más de 2.600 serotipos descritos hasta la fecha?

Aquellos serotipos causantes de enfermedad en animales de sangre caliente pertenecen a la sub-especie enterica ${ }^{9}$, y comparten más de $90 \%$ de su ADN. Sin embargo, tienen diferencias importantes tales como el rango de hospederos a los cuales infectan y los síntomas clínicos de la enfermedad que producen ${ }^{10}$. Es así como S. enterica serovar Enteritidis ( $S$. Enteritidis) y $S$. enterica serovar Typhimuirum ( $S$. Typhimurium) poseen un amplio rango de hospederos y la mayoría de las veces generan una enfermedad gastrointestinal en el ser humano, infección sistémica en el ratón y un cuadro crónico asintomático en aves. Otros serovares en cambio, tienen una alta especificidad de hospedero y generalmente causan la enfermedad en una única especie animal, como es el caso de $S$. enterica serovar Typhi ( $S$. Typhi), que afecta sólo al ser humano generando una enfermedad sistémica denominada fiebre tifoidea ${ }^{11}$.

Salmonella se transmite por la ruta fecal-oral, ya sea directamente, o bien indirectamente, a través de los alimentos. Es capaz de sobrevivir la acidez del estómago y la alta osmolaridad del intestino delgado, induce su internalización por las células epiteliales intestinales del íleon y resiste la fagocitosis mediada por las células dendríticas y macrófagos, logrando colonizar el tejido linfoide subyacente y los ganglios linfáticos mesentéricos ${ }^{12,13}$. Los macrófagos se consideran las células blanco de la infección, ya que la bacteria se establece en un compartimiento ácido conocido como la vacuola que contiene a Salmonella, donde es capaz de evadir la actividad lítica de los componentes lisosomales, multiplicarse y propagarse 
en el tejido intestinal o en el resto del organismo de su hospedero cuando se establece una infección local o sistémica, respectivamente ${ }^{12}$.

En Chile, la salmonelosis humana se encuentra dentro de las enfermedades de notificación obligatoria, siendo el Instituto de Salud Pública (ISP) el laboratorio nacional de referencia para su diagnóstico. El ISP realiza la confirmación de especie mediante caracterización bioquímica y tipifica serológicamente los aislados según el esquema de Kauffmann-White ${ }^{14}$.

Sin embargo, en un bajo porcentaje de los brotes se logra identificar el agente causal, correspondiendo en su mayoría a los serotipos Enteritidis y Typhimurium ${ }^{14}$, en concordancia con los datos mundiales ${ }^{15}$. Esta situación se ha venido repitiendo en los últimos años y demuestra la importancia del agente entre las enfermedades transmitidas por alimentos (ETAs) registradas, pero también da cuenta de la insuficiente cobertura diagnóstica en la investigación de enfermedades asociadas al consumo de alimentos en el país.

En las poblaciones animales, la información es aún más incompleta, aunque desde el año 2009 existe un Programa Nacional de Control de Salmonella spp., a cargo del Servicio Agrícola y Ganadero (SAG). En este programa se monitorean y recolectan datos desde establecimientos comerciales de aves, de los serotipos prioritarios para la Unión Europea, esto es $S$. Enteritidis, $S$. Typhimurium, $S$. Hadar, $S$. Virchow y $S$. Infantis ${ }^{16}$. A pesar que se ha reportado una baja o nula detección de los serotipos Enteritidis y Typhimurium ( $0 \%$ y $1,23 \%$, respectivamente), la vigilancia sólo es obligatoria en planteles productores de carne de pollo y pavo adscritos al Programa de Planteles Animales bajo Certificación Oficial (PABCO) y planteles que exporten aves o sus productos, por lo que esos resultados no representan la situación epidemiológica del país. Sumando a lo anterior, no existen reportes sobre la infección por Salmonella en las explotaciones no comerciales, en que miles de pequeños agricultores crían aves de corral y otros animales domésticos para el autoconsumo o la comercialización a baja escala, y generalmente no tienen adecuadas medidas de bioseguridad ni políticas de control. Es probable que los brotes de ETAs en estas personas no se diagnostican ni se denuncian, debido al contexto familiar y pequeño número de personas afectadas en cada evento ${ }^{17}$.

A pesar de las campañas de higiene y educación en la población y de las normativas sanitarias existentes para la producción de alimentos inocuos, los brotes de ETA por Salmonella no han disminuido significativamente en Chile, y más aún, algunos serotipos han incrementado su importancia relativa ${ }^{14}$. Esto lleva a suponer la existencia de otros ciclos de transmisión, que probablemente no están siendo abordados adecuadamente por la autoridad sanitaria ni han sido caracterizados por los grupos de investigación, resultando en las altas tasas de incidencia de este agente.

¿Qué factores pueden explicar la emergencia y amplia distribución de $S$. enterica en Chile? El objetivo de esta revisión es abordar aquellos factores que podrían ser relevantes en relación a este tema, como una propuesta que sirva para estimular el desarrollo de futuras líneas de investigación y de esta forma generar el conocimiento necesario para la implementación, con base en la evidencia científica, de medidas efectivas en la prevención de la salmonelosis humana y animal.

\section{Los factores que participan en la emergencia de S. enterica}

El comportamiento de cada enfermedad en la población depende de múltiples factores, que epidemiológicamente se han clasificado en tres grandes grupos de acuerdo a su origen: factores del propio agente biológico, del medio ambiente y del hospedero ${ }^{18}$. Es la inter-relación temporal y geográfica entre estos elementos lo que explican la historia natural de cualquier enfermedad, y las salmonelosis no son la excepción.

\section{Los factores del agente}

Probablemente las funciones de virulencia y supervivencia codificadas en las islas de patogenicidad, la capacidad de formar biopelículas y el progresivo aumento de la resistencia contra los antimicrobianos, constituyen los elementos que le permiten a Salmonella su amplia diseminación y distribución en la naturaleza, transformándose en una amenaza para la salud pública que persiste en el ambiente, en los alimentos y en un amplio rango de hospederos.

\section{Las islas de patogenicidad}

El genoma de las bacterias entéricas se encuentra sometido a una intensa presión selectiva debido a factores que incluyen la competencia con la microbiota normal, la adquisición de nutrientes en el organismo y en el ambiente, y la interacción con la respuesta inmune del hospedero. Esto se traduce en la presencia de genes y bloques de genes que tienen una constitución diferente respecto del resto del cromosoma, y que participan mejorando la adaptación o modificando la patogenicidad de las bacterias ${ }^{19}$. En general, estos segmentos se conocen como islas genómicas, pero cuando codifican funciones de virulencia se les denomina islas de patogenicidad (SPI, del inglés "Salmonella pathogenicity islands" ${ }^{20}$. Las islas de patogenicidad constituyen importantes elementos en la evolución de bacterias patógenas dado que, en un simple 
paso, un organismo o bacteria benigna puede transformarse en un patógeno ${ }^{21,22}$. Estas regiones cromosómicas están relacionadas con virulencia y generalmente se encuentran ausentes en cepas no virulentas de la misma especie ${ }^{22}$.

Por su mayor tasa de variabilidad genética, las SPI determinan estrategias diferenciales entre distintos serotipos de $S$. enterica, modificando la epidemiología y patogénesis de las infecciones que producen ${ }^{9}$. Aunque se han descrito más de 20 SPI, sólo cinco de ellas están presentes en todos los serotipos de la especie, y, por lo tanto, existen mecanismos comunes y otros específicos que participan en el proceso infeccioso, ya sea en el intestino o durante la invasión de los tejidos de su hospedero ${ }^{23,24}$.

A pesar de que las SPIs son diversas en cuanto a su estructura y función, se pueden encontrar ciertas propiedades comunes. Se caracterizan por poseer un porcentaje de los nucleótidos $\mathrm{G}+\mathrm{C}$ distinto al promedio del resto del genoma de la bacteria, se encuentran frecuentemente adyacentes a genes de ARNt, y por lo general están delimitadas por secuencias repetidas de nucleótidos. Estas características concuerdan con su probable adquisición mediante transferencia horizontal de genes, aunque su origen, así como los mecanismos de transferencias no han sido clarificados para muchas de ellas ${ }^{23,25}$.

Las islas más estudiadas y caracterizadas son SPI-1 y SPI-2. Estas son fundamentales para el éxito de la infección ya que están relacionadas con la invasión de células epiteliales y supervivencia de Salmonella en el interior de macrófagos ${ }^{25,26}$. La principal característica de ambas islas es que codifican sistemas de secreción tipo 3 (SSTT) y las denominadas proteínas efectoras de dichos sistemas ${ }^{27}$. Los SSTT son estructuras con analogía a jeringas mediante las cuales se secretan las proteínas efectoras que ayudan al patógeno a invadir células humanas o de animales ${ }^{27}$. SPI-1 está relacionada con la invasión de células no fagocíticas mediante un proceso que involucra la modificación del citoesqueleto o "ruffling". También participa en el proceso de replicación intracelular, interviene con la respuesta inmune del hospedero y algunos genes se relacionan con la formación de biopelículas ${ }^{20,25,27-29}$. Dada la importancia de SPI-1 en el ciclo infectante de Salmonella, mutantes en esta isla han sido explorados como cepas atenuadas candidatas a vacunas ${ }^{20}$. SPI-2 codifica alrededor de 32 genes, entre los que destacan un SSTT y sus proteínas efectoras que regulan la replicación y supervivencia de Salmonella al interior de células epiteliales y fagocíticas, permitiendo que la bacteria pueda generar una infección sistémica ${ }^{25-27,30}$. Al igual que con SPI-1, mutantes en esta isla son candidatos a cepas atenuadas como vacunas ${ }^{30}$.

Los mecanismos por los que Salmonella genera una infección no sólo requieren la presencia de estas islas por separado, sino que debe existir una intrincada comunicación y expresión regulada de sus genes para que el proceso infeccioso sea exitoso.

\section{Las biopelículas}

Uno de los factores que favorecen la persistencia de Salmonella en variados ambientes es su capacidad de formar biopelículas sobre diferentes tipos de superficies, inertes y vivas. Esta habilidad contribuye a su supervivencia tanto en ambientes hostiles como también en condiciones favorables para su desarrollo ${ }^{31}$.

Las biopelículas son definidas como una agregación de microorganismos rodeadas por una matriz que está compuesta por una mezcla de sustancias extracelulares poliméricas (SEP). Esta matriz está formada por $97 \%$ de agua y el resto es principalmente exopolisacáridos, proteínas, lipopolisacáridos, ácidos nucleicos y ácidos grasos. Las biopelículas pueden formarse sobre una gran variedad de superficies (plástico, vidrio, gomas, acero inoxidable, etc.) y son los biopolímeros extracelulares los responsables de la adhesión de las células a las mismas ${ }^{32}$. En algunos serotipos de $S$. enterica como $S$. Typhimurium, las SEP que contribuyen a la formación de biopelículas incluye fimbrias, celulosa, polisacáridos capsulares y otros polisacáridos como el lipopolisacárido (LPS) ${ }^{33}$.

Las SEP protegen a los microorganismos contra agentes antimicrobianos, previenen el acceso de biocidas, quelantes, toxinas, evitan la deshidratación, refuerzan la resistencia de la biopelícula al estrés ambiental y permiten a los microorganismos capturar los nutrientes, siendo muy difíciles de eliminar. Debido a estas características, las biopelículas modifican aspectos esenciales de la capacidad infectante bacteriana y han estado aumentando progresivamente su impacto en la generación de problemas de salud serios y persistentes ${ }^{34,35}$.

La formación de biopelículas le permitiría a Salmonella sobrevivir a largo plazo en el entorno de las granjas de aves de corral y contaminar la carne y huevos o alimentos derivados, los que siguen siendo vehículos principales de los brotes de salmonelosis de origen alimentario ${ }^{36}$. Adicionalmente se han identificado biopelículas naturales de Salmonella en tomates frescos, zanahorias, hongos, brotes de alfalfa, etc. Esta capacidad representa un problema sustancial en la seguridad alimentaria ya que actualmente no hay un desinfectante que pueda alcanzar una reducción importante de las biopelículas formadas por patógenos en productos frescos de origen vegetal, lo que constituye un riesgo importante para la salud pública ${ }^{37}$.

A nivel industrial la formación de biopelículas en superficies que están en contacto con alimentos es un reservorio de bacterias patógenas, incrementando el riesgo de contaminación microbiana ${ }^{38}$. Se ha demostrado que las aves de corral contaminadas con Salmonella aumentan entre 15 y $30 \%$ después del procesamiento, como consecuencia de la presencia de bacterias en las superficies en contacto con alimentos ${ }^{38}$. Pero una de las grandes preocupaciones en la industria alimentaria es que en las infecciones asociadas a biopelículas hay una alta 
tolerancia a las condiciones de estrés y un aumento del potencial de virulencia de las bacterias ${ }^{39}$. Al respecto, se ha observado que el uso de concentraciones sub-letales de antimicrobianos o la exposición a condiciones estresantes sub-letales, induce a un incremento en la virulencia de las bacterias sobrevivientes (mayor capacidad invasora a células epiteliales humanas e inducción de apoptosis en macrófagos), teniendo éstas un alto potencial infeccioso en caso de entrar en contacto con un hospedero ${ }^{40}$. En ensayos de supervivencia en $S$. Typhimurium, se observó que la respuesta molecular al estrés juega un papel importante en la patogenicidad, ya que la formación de biopelículas aumentó entre 89 y 100\% después de la exposición a concentraciones sub-letales de polifenoles, permitiendo el incremento de la resistencia a antimicrobianos y su virulencia $^{39}$. La explicación a este comportamiento es un aumento en la expresión de genes involucrados en adhesión a células epiteliales, virulencia y eflujo de sustancias antimicrobianas. Adicionalmente, en este trabajo se determinó que el regulador $\mathrm{CsgD}$, involucrado en la formación de biopelículas, también controla la expresión del gen $a d r A$, involucrado en la virulencia de esta bacteria ${ }^{39}$.

En $S$. Enteritidis también se ha observado que la expresión de genes relacionados con virulencia puede alterarse por la exposición a estrés sub-letal, lo que se traduce en una disminución de la dosis infectante de la bacteria ${ }^{41}$. Son precisamente las células inmersas en biopelículas las que están expuestas a concentraciones sub-letales de antimicrobianos y agentes inductores de estrés, y por esta razón son las células que podrían expresar un mayor potencial de virulencia.

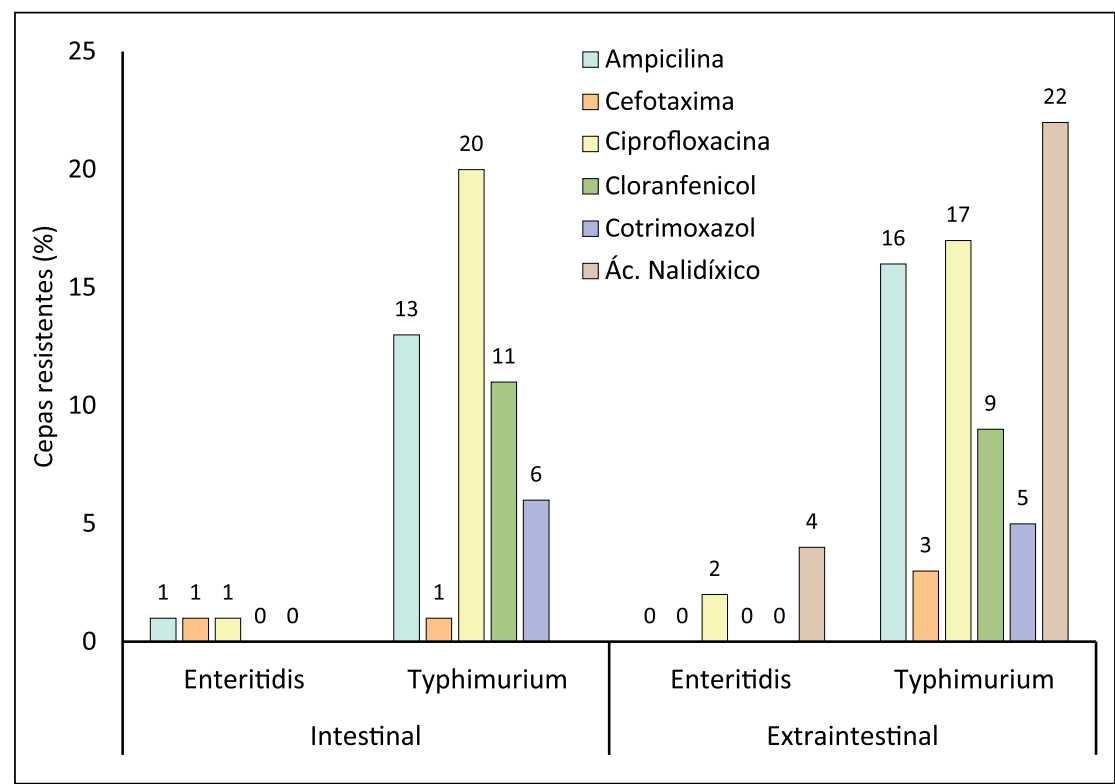

Figura 1. Porcentajes (\%) de cepas resistentes de Salmonella enterica aisladas desde personas en Chile entre los años 2009-2013. Los datos se muestran para los serotipos Enteritidis y Typhimurium, según origen intestinal o extra-intestinal de los aislados ${ }^{14}$
De este modo, los factores que inducen o previenen la formación de biopelículas son actualmente temas de interés para la industria animal, la autoridad sanitaria y los propios consumidores ${ }^{38}$. Es por ello que en $S$. enterica se han realizado muchos trabajos de investigación buscando las señales ambientales que regulan la formación de biopelículas y se han descrito algunas vías regulatorias involucradas en la formación de las mismas ${ }^{42}$. Sin embargo, los hallazgos que se describen son muy dependientes de la cepa y del serotipo bacteriano, por lo que se debe ajustar la implementación de medidas de control, en base a la realidad microbiológica de cada industria, región o país ${ }^{31}$.

\section{La resistencia antimicrobiana}

La resistencia antimicrobiana es considerada una amenaza creciente globalmente debido a la gran diversidad y volumen de antimicrobianos prescritos en medicina humana y animal, lo que ha facilitado la selección y dispersión de patógenos resistentes a estos fármacos (WHO, 2014 ${ }^{43}$ ). Diversos factores han contribuido a este fenómeno que va progresivamente afectando las alternativas terapéuticas, con un impacto mayor en enfermedades potencialmente letales. La prescripción muchas veces innecesaria de antimicrobianos por parte de los médicos, la automedicación, y el mal uso de estos fármacos como promotores del crecimiento en la industria animal son causas principales del problema. Por ello, la OMS ha definido una lista de antimicrobianos denominados críticamente importantes, que deben ser utilizados prudente y preferentemente con ciertos patógenos y bajo ciertas condiciones específicas ${ }^{43}$.

Salmonella es considerada una bacteria que se transmite frecuentemente a la población humana desde los animales y, por lo tanto, tiene el riesgo asociado de transferir determinantes de resistencia que se han seleccionado a través de las mencionadas malas prácticas veterinarias ${ }^{43}$. Por este motivo, el Centro de Control de Enfermedades de E.U.A. (CDC) en su último reporte de amenazas identifica a Salmonella como una amenaza seria, que requiere de mayor investigación, vigilancia y monitoreo ${ }^{44}$.

Ya que generalmente la salmonelosis en seres humanos se presenta como una gastroenteritis autolimitada, los antimicrobianos se recomiendan principalmente en cuadros extra-intestinales invasores, que en Chile corresponden a $10 \%$ de los casos diagnosticados, aproximadamente ${ }^{14}$. En relación a la resistencia antimicrobiana, el CDC identifica a ceftriaxona y ciprofloxacina como las resistencias de preocupación, pero además el surgimiento de cepas multiresistentes a cinco o más clases de antimicrobianos ${ }^{44}$. En Chile, la vigilancia del ISP sugiere la presencia de bajos niveles de resistencia con el serotipo Enteritidis (menos de $2 \%$ ), siendo de mayor relevancia la situación observada con Typhimurium, que presenta más de $15 \%$ de cepas resistentes a ciprofloxacina y ácido nalidíxico, especialmente en los aislados extra-intestinales (Figura 1) ${ }^{14}$. Esta 
situación es concordante con lo que se ha reportado en otros países, en que se han verificado diferencias entre serotipos y se ha establecido que $S$. Enteritidis posee, en general, menores niveles de resistencia antimicrobiana ${ }^{10}$.

Sin embargo, estudios en cepas de $S$. enterica aisladas desde hospederos animales en Chile, correspondientes principalmente al serotipo Enteritidis, sugieren que los niveles de resistencia son significativamente mayores, siendo tetraciclina aquel antimicrobiano en que se observa el mayor porcentaje de cepas resistentes $(70 \%)^{45-47}$. Esto implica que existen diferencias entre hospederos y que los animales, a través de las bacterias que los colonizan, están expresando el impacto antropogénico en el medio ambiente, determinado en este caso por la dispersión de residuos de antimicrobianos o directamente de bacterias resistentes ${ }^{48,49}$. Otro factor que puede explicar estos hallazgos, es la presencia de los denominados plasmidios de virulencia-resistencia en Salmonella, que codifican, tanto factores de resistencia antimicrobiana como factores de virulencia y, por lo tanto, pueden existir procesos de co-selección de estos determinantes, incluso en ausencia de antimicrobianos ${ }^{50,51}$. En cualquiera de los casos, la persistencia ambiental de bacterias multi-resistentes constituye un riesgo para la salud pública y para la salud de los animales ${ }^{52}$, y resulta imperiosa la investigación para caracterizar este "resistoma" ambiental que permita la implementación de medidas de prevención y vigilancia, y que impulse la evaluación de estrategias alternativas al uso de antimicrobianos en la industria, como por ejemplo la aplicación de extractos naturales o la fagoterapia ${ }^{53,54}$, entre otros.

\section{Los factores ambientales}

Se ha determinado que la mayoría de las EE se relacionan con patógenos que circulan en la fauna silvestre ${ }^{1}$, que se transmiten a la especie humana o a los animales domésticos debido a la intervención y modificación de hábitats naturales para el desarrollo de actividades agrícolas, forestales o para la construcción de casas u otro tipo de asentamientos humanos ${ }^{55}$. Adicionalmente, el cambio climático, los nuevos sistemas de producción animal y la polución constituyen otras variables ambientales que han facilitado el surgimiento de $\mathrm{EE}^{55}$.

En el caso de Salmonella, es probable que todos estos factores puedan modificar la transmisión y la epidemiología de la infección, pero con algunos de ellos existen evidencias que dan cuenta de su importancia.

\section{La fauna silvestre}

En cuanto a la fauna silvestre, diversos reportes sugieren que la infección es prevalente en animales de ambientes acuáticos, incluyendo aves y mamíferos ${ }^{56,57}$, aunque se desconoce el efecto ecológico de la infección en estas poblaciones. De ellos, el grupo con un mayor número de evidencias como portadores de $S$. enterica son las aves, debido a que estos animales han recibido el interés de los organismos sanitarios por su capacidad para difundir patógenos zoonóticos altamente transmisibles ${ }^{58,59}$. Su papel como reservorios es crucial debido a eventos de migración periódicos que caracterizan algunas de estas especies, con la potencialidad para adquirir y transmitir patógenos sobre extensas zonas geográficas, en breves períodos de tiempo e interactuando con múltiples especies animales a lo largo de sus rutas migratorias o en su lugares de destino ${ }^{59}$.

Los serotipos de Salmonella más frecuentemente aislados desde las aves silvestres (principalmente aves acuáticas) son Typhimurium y Enteritidis ${ }^{59-61}$, existiendo reportes que sugieren una transmisión de la bacteria entre estas aves, los seres humanos y otros animales ${ }^{47,62-64}$. Aunque en la mayoría de los casos la infección de las aves es asintomática, estos serotipos también se han asociado a brotes de enfermedades y alta mortalidad en los animales ${ }^{59,65-68}$.

En Chile, estudios recientes en aves acuáticas tanto residentes como migratorias, han confirmado la presencia de $S$. enterica, siendo $S$. Enteritidis el serotipo de mayor frecuencia con más de $50 \%$ de los aislados ${ }^{45,46,69,70}$. Además, se reporta en estos animales la existencia de cepas multi-resistentes a los antimicrobianos, así como el aislamiento de $S$. Dublin, un serotipo zoonótico relacionado frecuentemente al ganado doméstico ${ }^{71}$. Estos hallazgos, junto con estudios que muestran cepas genotípicamente idénticas, aunque provenientes de distintos hospederos, sugieren que las aves acuáticas son reservorios naturales de S. enterica, que mediante eventos de transmisión interespecies, participan en la epidemiología de la infección, tanto en seres humanos como en la fauna doméstica y silvestre de nuestra región ${ }^{47}$.

En esta perspectiva, resulta interesante que más de $30 \%$ de los brotes de ETA confirmados en Chile se asocian al consumo de pescados y productos de la pesca ${ }^{72,73}$. Entonces la implicancia de las aves en la contaminación de estos alimentos, el papel de otros animales acuáticos en que también se ha detectado infección con serotipos zoonóticos de Salmonella ${ }^{46,74}$ y el efecto del ser humano en la dispersión de patógenos y genes de resistencia a los antimicrobianos, podrían explicar el riesgo de infección a partir del consumo de productos del mar y contribuir tanto a los crecientes niveles de incidencia, como a la emergencia de nuevas cepas patógenas de Salmonella.

\section{La contaminación}

Con efectos sobre los ecosistemas, la biodiversidad, la proliferación de vectores y la dispersión de agentes por el aire, la tierra y el agua, la contaminación representa uno de los principales desafíos en la preservación del entorno natural, especialmente en centro urbanos con altas 
densidades poblacionales. La contaminación se establece no sólo como un elemento que amenaza los equilibrios ecológicos, sino que también facilita el contacto de animales silvestres con agentes biológicos o productos tóxicos de origen humano, determinando un riesgo mutuo en la transmisión de enfermedades o en la susceptibilidad hacia ellas ${ }^{55,75}$. En el caso de Salmonella, la detección de diversos serotipos y múltiples aislados en aguas y en la fauna silvestre pueden ser una expresión de este problema de contaminación ${ }^{76,77}$. La correlación positiva entre los coliformes fecales con la detección de otros patógenos transmitidos por el agua, incluyendo a Salmonella ${ }^{78}$, es una evidencia que podría ser investigada y validada en distintos escenarios epidemiológicos, para ser utilizada en la implementación de programas de vigilancia y/o fiscalización de efluentes en zonas de riesgo de contaminación.

El impacto en el ambiente puede ser temporal, cuando se asocia a derrames accidentales de aguas servidas, inundaciones o escorrentías de aguas lluvia, o bien persistente cuando existe descarga continua de aguas servidas o desecho, una gran concentración de animales silvestres $\mathrm{u}$ otros reservorios ambientales presentes. En Chile, la detección de cepas de Salmonella multi-resistentes a los antimicrobianos en animales silvestres, es la evidencia que podría ser utilizada como un indicador al respecto ${ }^{45,46}$, aunque al igual que con los coliformes fecales, también se requiere una validación de esta relación entre los niveles de polución ambiental y los hallazgos en la fauna que habita en estos lugares. El mayor riesgo para el ser humano se presenta por el contacto directo con cursos de agua, por el consumo de alimentos vegetales cultivados con aguas contaminadas, o por el consumo de alimentos del mar extraídos en cercanías a drenajes de aguas servidas ${ }^{79}$.

\section{Los cambios en la industria pecuaria}

Este factor se manifiesta con la intensificación de los procesos productivos, en que un mayor número de animales se cría en espacios relativamente acotados, y donde el estrés productivo predispone a estos individuos para la infección y transmisión de agentes biológicos. Sin embargo, otros factores que vinculan a la industria pecuaria con la emergencia de enfermedades son la menor variabilidad genética de los animales, el uso excesivo de antimicrobianos, los movimientos de personas y animales vinculados a estos establecimientos, los cambios drásticos en la alimentación y la contaminación de cursos de agua con los grandes volúmenes de desechos biológicos propios de los planteles industriales ${ }^{55}$. Salmonella es capaz de infectar prácticamente a todas las especies animales productivas ${ }^{10,11}$, siendo en planteles intensivos donde existe un mayor riesgo de transmisión debido a la alta concentración de hospederos susceptibles, que además defecan y se alimentan en el mismo entorno. De hecho, en nuestro país, el cambio epidemiológico de
Salmonella a principios de los 90 se debió en parte a la mejor higienización y disposición de las aguas servidas, logrando una baja significativa en la incidencia de $S$. Typhi, pero también a los cambios introducidos en la producción y comercialización de productos de origen animal, particularmente aviar, que explican el alza de las ETAs provocadas por $S$. Enteritidis, situación que se mantiene hasta el día de hoy ${ }^{36,80}$.

La alta tasa de contactos que implica la crianza intensiva de animales, facilita la transmisión de agentes biológicos y la selección de cepas de mayor virulencia ${ }^{81}$. En un trabajo reciente, se detectó efectivamente que cepas de $S$. Enteritidis aisladas desde aves comerciales tienen una virulencia potencial mayor que cepas aisladas desde aves silvestres o desde personas, ya que resisten mejor a condiciones de estrés asociadas a la patogenicidad tales como $\mathrm{pH}$ ácido, exposición a radicales libres y hambruna ${ }^{47}$. Por lo tanto, los cambios en la industria animal predisponen a la emergencia de cepas de mayor virulencia, siendo fundamental la aplicación de medidas de bioseguridad que impidan el ingreso y la propagación de agentes patógenos en un ambiente de alta densidad de individuos susceptibles.

\section{Los factores del hospedero humano}

En los últimos años el ser humano ha experimentado cambios socioculturales significativos, asociados a la globalización, al gran tamaño de la población y a su concentración en zonas urbanas. Estos cambios se reflejan en el gran intercambio de productos y subproductos alimenticios, así como en los viajes de personas alrededor del planeta, muchas veces con fines ecoturísticos donde aumenta el riesgo de exposición a vectores, hospederos y patógenos de otras zonas geográficas. Pero también la masificación de la crianza de animales de compañía y animales exóticos, y el consumo de alimentos exóticos o crudos son factores predisponentes que se han popularizado recientemente, especialmente en las culturas occidentales.

\section{Globalización}

La globalización, como determinante de la emergencia de patógenos, se traduce en la comercialización de animales, vegetales y alimentos para consumo humano y animal entre distintas zonas geográficas, que se han vinculado con diversas ETAs, entre ellas Salmonella ${ }^{82,83}$.

Chile importa cientos de millones de dólares en este tipo de productos (Servicio Nacional de Aduanas, http:// www.aduana.cl), por lo que el riesgo existe y podría generar un número de brotes que, debido a la escasa identificación del origen de estos casos, podría ser un componente sub-diagnosticado en la epidemiología de la infección. 


\section{Animales de compañía}

No existen antecedentes respecto de la presencia de Salmonella en mascotas en Chile, y en general, pocos esfuerzos se han realizado para dilucidar este riesgo sanitario a nivel mundial. Sin embargo, perros y gatos son hospederos de la infección, y se han realizado algunos estudios en poblaciones animales específicas de países desarrollados que establecen niveles de infección entre 1 y $5 \%$ de los animales muestreados ${ }^{84-86}$.

Debido al más de medio millón de perros que deambulan libremente en zonas urbanas y rurales ${ }^{87}$, y al estrecho contacto que existe entre muchos de estos animales y sus dueños, es que se transforma en un factor de riesgo en Chile que merece una alta prioridad en su caracterización. Además, la masificación en el uso de alimentos comerciales para la alimentación de las mascotas, que también podrían ser una fuente de infección en ellos ${ }^{88}$, hace más compleja la caracterización del potencial problema sanitario representado por estos animales.

Las infecciones de personas adquiridas a partir del contacto directo con animales de compañía, podrían ser significativas, pero pasar frecuentemente desapercibidas para los sistemas de vigilancia, debido a que se tratarían de pequeños brotes, radicados entre los propietarios de estos animales, que escasamente se informan a los servicios de salud y menos aún se reportan a través de publicaciones científicas ${ }^{17}$.

La relación epidemiológica de estas especies como fuente de brotes humanos normalmente se ha basado en la serotipificación o evidencias circunstanciales ${ }^{17}$, lo que sugiere la necesidad de una vigilancia activa con el apoyo de métodos de caracterización molecular, dirigida no sólo a brotes de ETAs que involucran a muchas personas, sino que también hacia aquellos casos más acotados, tanto en ambientes urbanos como rurales. En especial los sistemas productivos de traspatio, ampliamente distribuidos en países en desarrollo, constituyen un escenario epidemiológico prácticamente inexplorado que podría ser motivo frecuente de ETAs con diversos agentes etiológicos ${ }^{89}$.

\section{Animales exóticos}

La crianza de animales exóticos de compañía constituye una tendencia creciente tanto en países desarrollados como en vías de desarrollo. El comercio legal e ilegal de estos animales, y su capacidad de infectarse con agentes biológicos propios de su entorno natural, transforman a estas especies en riesgos potenciales de $\mathrm{EE}^{90}$. Aves psitácidas, reptiles y roedores se encuentran entre los tipos de animales que frecuentemente se asocian a casos clínicos en sus dueños. Recientemente se han reportado brotes de Salmonella en personas producto de la crianza de tortugas en múltiples estados de E.U.A. ${ }^{91}$. En Chile, un estudio retrospectivo realizado por profesionales del SAG en reptiles importados y mantenidos en cuarentena entre los años 1997 y 2008, determinó que 70\% de ellos era portador de Salmonella, con un alto porcentaje (43\%) de serotipos que no se habían descrito previamente en nuestro país ${ }^{92}$. Esta condición implica un alto riesgo de contagio para las personas que tienen contacto directo e indirecto con estos animales, y particularmente para niños bajo 5 años de edad, que son más susceptibles a la infección. En concordancia con esta realidad, un estudio nacional ha reportado cuadros de salmonelosis en tres lactantes como consecuencia de la crianza de tortugas de agua adquiridas previamente por sus familias en ferias libres $^{93}$. Tanto la manipulación directa de los animales, como la contaminación cruzada de sus alimentos son las causas más probables de la transmisión, que en algunos casos también afectó a otras personas expuestas en las mismas casas.

Las mascotas exóticas son, por lo tanto, un riesgo para la emergencia de serotipos zoonóticos de Salmonella y probablemente de otros agentes biológicos, por lo que no debiera promoverse la comercialización y crianza de estos animales.

\section{Consumo de alimentos exóticos}

El consumo de alimentos exóticos, ya sea por la caza directa o la comercialización en mercados y restaurantes, se ha asociado frecuentemente al riesgo de emergencias, especialmente en Asia y África. Asimismo, en naciones industrializadas o en vías de desarrollo, se ha hecho más frecuente este mismo tipo de prácticas, ya sea por la demanda de las personas y la imposición de ciertas modas, o bien por los flujos migratorios que, junto con las personas, determina también la migración de la cultura y costumbres propias de estas poblaciones ${ }^{90}$. Es probablemente el consumo de alimentos crudos el factor determinante en la incidencia de brotes relacionados a este factor, existiendo el reporte de casos masivos y en amplias zonas geográficas ${ }^{94}$. En Chile, la infección con Salmonella ha estado relacionada al consumo de comida japonesa o "sushi", donde deficiencias en la cadena de frío y la contaminación cruzada de alimentos ha provocado cuadros de gravedad en personas de diversos grupos de riesgo, incluyendo lactantes y mujeres embarazadas ${ }^{95}$. Es de preocupación la probable sub-notificación de casos que, por distintos factores, impiden o retrasan el diagnóstico y el despliegue oportuno de los sistemas de monitoreo y vigilancia.

\section{Desafíos para el futuro}

En términos generales, todos los factores identificados en esta revisión contienen vacíos de conocimiento, han sido sustentados por evidencias puntuales o parciales y, por tanto, se constituyen en desafíos para la investigación 
Figura 2. Factores predisponentes para la emergencia de $S$. enterica en Chile.

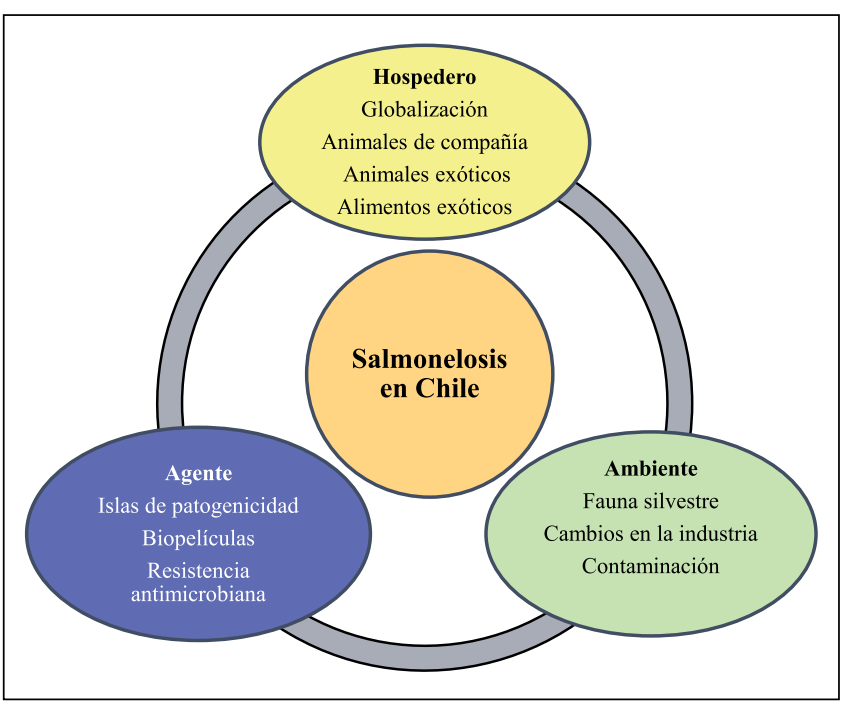

a futuro. El cuadro general de estos factores predisponentes (Figura 2) tiene valor cualitativo por cuanto reconoce su existencia en nuestro escenario epidemiológico, pero carece de dos aspectos fundamentales: la importancia relativa de cada uno de ellos (para cada serotipo de Salmonella) y las estrategias para su control y prevención.

El primer elemento debe surgir de la investigación científica promovida a través de la coordinación de las instituciones vinculadas con la salud y alimentación de personas y animales, incluyendo a la industria, el Ministerio de Salud, el Ministerio de Agricultura y las Universidades. Se trata de un tema complejo y multifactorial que requiere la comunicación entre estas instancias, situación que ha sido reconocida para todas las EE y que ha motivado a las autoridades sanitarias internacionales en el establecimiento del concepto de una salud (http:// www.onehealthinitiative.com/). Es la evidencia científica lo que permitirá seleccionar y evaluar estrategias de acción en pos del control y la prevención, con lo que sumamos el segundo elemento faltante de nuestra propuesta.

Si hubiese que proponer un modelo a seguir, es quizás la Unión Europea el mejor referente, ya que ha logrado avances efectivos en disminuir la incidencia de salmonelosis en las personas. Bajo una estrategia denominada "de la granja al tenedor" ("from farm to fork"), existe una coordinación inter-institucional para la comunicación rápida y efectiva del riesgo, estableciendo bases de datos centralizadas cuyo análisis permite la generación de recomendaciones específicas basadas en evidencia científica $^{8,96}$. Se trabaja precisamente bajo el enfoque integrado de una salud.

En Chile ya existen instancias para la vigilancia y la investigación que reconocen a la salmonelosis como un problema sanitario, y además existe la decisión política de mejorar la calidad e inocuidad de los alimentos, tanto en el contexto de metas sanitarias como de metas comerciales $^{97,98}$. Entonces son la actividad integrada entre las instituciones y la estandarización de las metodologías de trabajo los desafíos actuales más importantes para nuestro país, requiriéndose el marco normativo-procedimental y el financiamiento para concretar lo que ya es una necesidad asumida por todos las partes. La autoridad sanitaria y animal, las universidades y la industria deben establecer mecanismos de acción e investigación consistentes, que permitan la obtención estructurada de datos. La información de calidad es clave para la toma de decisiones basadas en evidencia científica.

Finalmente, desde la perspectiva de los factores predisponentes mencionados en esta revisión, otros grandes desafíos en el conocimiento de la enfermedad en Chile son:

- Caracterizar, microbiológica y epidemiológicamente, los factores de virulencia y determinantes de resistencia de las cepas de Salmonella con mayor impacto en la salud pública y animal del país.

- Implementar sistemas de vigilancia de Salmonella en fauna silvestre y muestras ambientales, que identifique el efecto de la actividad antropogénica y anticipe el surgimiento de nuevas cepas o serotipos con potencial impacto sanitario.

- Identificar y evaluar estrategias de control y prevención a nivel de la industria de alimentos, incluyendo programas de educación de los propietarios de animales y agricultores, medidas de bioseguridad predial, la vacunación y otras medidas complementarias.

- Identificar la asociación causa-efecto de la infección por Salmonella en la población humana y animal, fortaleciendo los sistemas de vigilancia epidemiológica antes y después de un brote. Se requiere implementar la epidemiología molecular como herramienta de rutina para determinar la relación de aislados provenientes de casos clínicos, alimentos, animales y el ambiente, a fin de identificar las vías de transmisión más importantes de la bacteria.

- Se debe mejorar la notificación de casos clínicos y el diagnóstico etiológico, en escenarios de pequeños y grandes brotes, para caracterizar el riesgo a distintas fuentes de infección.

\section{Resumen}

Salmonella enterica es uno de los principales patógenos transmitidos por los alimentos en el mundo, siendo la primera causa de brotes de intoxicación alimentaria en Chile. A pesar de todos los esfuerzos de control y prevención desplegados, la incidencia en las personas se ha mantenido alta, por lo que diversos factores podrían 
estar influenciando el comportamiento epidemiológico de esta infección. El objetivo de esta revisión es describir factores referidos tanto al agente biológico, al hospedero humano y al medio ambiente, que podrían tener mayor trascendencia en Chile. De esta forma, se infieren ámbitos prioritarios para la investigación de $S$. enterica, que permitan entender su dispersión en la naturaleza y su éxito como patógeno de un amplio rango de hospederos. A futuro, el mayor conocimiento de estos determinantes facilitará la implementación de estrategias de bioseguridad y vigilancia para la prevención de la enfermedad en las personas y en los animales.

\section{Referencias bibliográficas}

1.- Jones K E, Patel N G, Levy M A, Storeygard A, Balk D, Gittleman J L, et al. Global trends in emerging infectious diseases. Nature 2008; 451: 990-3.

2.- Woolhouse M E. Epidemiology: emerging diseases go global. Nature 2008; 451: 898-9.

3.- Morse S S, Mazet J A, Woolhouse M, Parrish C R, Carroll D, Karesh W B, et al. Prediction and prevention of the next pandemic zoonosis. Lancet 2012; 380: 1956-65.

4.- Schneider M C, Aguilera X P, Smith R M, Moynihan M J, Silva J B, Aldighieri S, et al. Importance of animal/human health interface in potential Public Health Emergencies of International Concern in the Americas. Rev Panam Salud Publica 2011; 29: 371-9.

5.- Cardoen S, Van Huffel X, Berkvens D, Quoilin S, Ducoffre G, Saegerman C, et al. Evidence-based semiquantitative methodology for prioritization of foodborne zoonoses. Foodborne Pathog Dis 2009; 6: 1083-96.

6.- Olea A, Díaz J, Fuentes R, Vaquero A, García M. Vigilancia de brotes de enfermedades transmitidas por alimentos en Chile. Rev Chilena Infectol 2012; 29: 504-10.

7.- CDC. Surveillance for foodborne disease outbreaks--United States, 2009-2010. MMWR Morb Mortal Wkly Rep. 2013; 62: 41-7.

8.- EFSA. The European Union summary report on trends and sources of zoonoses, zoonotic agents and food-borne outbreaks in 2013. EFSA J. 2015; 13: 3991.

9.- Amavisit P, Lightfoot D, Browning G F, Markham P F. Variation between pathogenic serovars within Salmonella pathogenicity islands. J Bacteriol 2003; 185: 3624-35.

10.- Andino A, Hanning I. Salmonella enterica: survival, colonization, and virulence differences among serovars. Scientific World J 2015; 2015: 520179.

11.- Uzzau S, Brown DJ, Wallis T, Rubino S, Leori G, Bernard S, et al. Host adapted serotypes of Salmonella enterica. Epidemiol Infect 2000; 125: 229-55.

12.- Cossart P, Sansonetti P J. Bacterial invasion: the paradigms of enteroinvasive pathogens. Science 2004; 304: 242-8.

13.- Dandekar T, Fieselmann A, Fischer E, Popp J, Hensel M, Noster J. Salmonella-how a metabolic generalist adopts an intracellular lifestyle during infection. Front Cell Infect Microbiol 2014; 4: 191.

14.- ISP. Vigilancia de laboratorio Salmonella spp. 2009-2014. Boletín 2014: Disponible en: http:// www.ispch.cl/content/21378.

15.- Hendriksen R S, Vieira A R, Karlsmose S, Lo Fo Wong D M, Jensen A B, Wegener H C, et al. Global monitoring of Salmonella serovar distribution from the World Health Organization Global Foodborne Infections Network Country Data Bank: results of quality assured laboratories from 2001 to 2007. Foodborne Pathog Dis 2011; 8: 887-900.

16.- SAG. Situación sanitaria animal de Chile 2014. Disponible en: http://www.sag.gob.cl/sites/ default/files/situacion_sanitaria_animal_2014. pdf.

17.- Hoelzer K, Moreno Switt A I, Wiedmann M. Animal contact as a source of human nontyphoidal salmonellosis. Vet Res 2011; 42: 34 .

18.- Thrusfield M. Veterinary Epidemiology: Wiley, 2007.

19.- Baker S, Dougan G. The genome of Salmonella enterica serovar Typhi. Clin Infect Dis. 2007; 45 Suppl 1: S29-33.

20.- Que F, Wu S, Huang R. Salmonella pathogenicity island 1(SPI-1) at work. Curr Microbiol 2013; 66: 582-7.

21.- Blanc-Potard A B, Solomon F, Kayser J, Groisman E A. The SPI-3 pathogenicity island of Salmonella enterica. J Bacteriol 1999; 181: 998-1004.

22.- Hacker J, Kaper J B. Pathogenicity islands and the evolution of microbes. Ann Rev Microbiol 2000; 54: 641-79.

23.- Schmidt H, Hensel M. Pathogenicity islands in bacterial pathogenesis. Clin Microbiol Rev 2004; 17: 14-56.

24.- Suez J, Porwollik S, Dagan A, Marzel A, Schorr Y I, Desai P T, et al. Virulence gene profiling and pathogenicity characterization of non-typhoidal Salmonella accounted for invasive disease in humans. PLoS One 2013; 8: e58449.

25.- Hensel M. Evolution of pathogenicity islands of Salmonella enterica. Int J Med Microbiol 2004; 294: 95-102.

26.- Altier C. Genetic and environmental control of Salmonella invasion. J Microbiol. 2005; 43 Spec No: 85-92.

27.- Figueira R, Holden D W. Functions of the Salmonella pathogenicity island 2 (SPI-2) type III secretion system effectors.

Microbiology 2012; 158: 1147-61.

28.- Hapfelmeier S, Ehrbar K, Stecher B, Barthel M, Kremer M, Hardt W D. Role of the Salmonella pathogenicity island 1 effector proteins SipA, SopB, SopE, and SopE2 in Salmonella enterica subspecies 1 serovar Typhimurium colitis in streptomycin-pretreated mice. Infect Immun 2004; 72: 795-809.

29.- Santos R L, Tsolis R M, Bäumler A J, Adams L G. Pathogenesis of Salmonellainduced enteritis. Braz J Med Biol Res 2003; 36: 3-12.

30.- Figueroa O, Verdugo R. Mecanismos moleculares de patogenicidad de Salmonella sp. Rev Latinoam Microbiol 2005. p. 25-42.

31.- Lianou A, Koutsoumanis K P. Strain variability of the biofilm-forming ability of Salmonella enterica under various environmental conditions. Int J Food Microbiol 2012; 160 : 171-8.

32.- Vu B, Chen M, Crawford R J, Ivanova E P. Bacterial extracellular polysaccharides involved in biofilm formation. Molecules 2009; 14: 2535-54.

33.- Dong H, Peng D, Jiao X, Zhang X, Geng S, Liu X. Roles of the spiA gene from Salmonella enteritidis in biofilm formation and virulence. Microbiology 2011; 157: 1798-805.

34.- Ledeboer N A, Jones B D. Exopolysaccharide sugars contribute to biofilm formation by Salmonella enterica serovar Typhimurium on HEp-2 cells and chicken intestinal epithelium. J Bacteriol 2005; 187: 3214-26.

35.- Bjarnsholt T, Alhede M, Eickhardt-Sørensen S $\mathrm{R}$, Moser $\mathrm{C}$, Kühl M, Jensen $\mathrm{P}$, et al. The in vivo biofilm. Trends Microbiol 2013; 21: 466-74.

36.- Fica A, Alexandre M, Prat S, Fernández A, Fernández J, Heitmann I. Cambios epidemiológicos de las salmonelosis en Chile. Desde Salmonella typhi a Salmonella enteritidis. Rev Chilena Infectol 2001; 18: 85-93.

37.- Jahid I K, Han N, Zhang C Y, Ha S D. Mixed culture biofilms of Salmonella Typhimurium and cultivable indigenous microorganisms on lettuce show enhanced resistance of their sessile cells to cold oxygen plasma. Food Microbiol 2015; 46: 383-94.

38.- Díez-García M, Capita R, Alonso-Calleja C. Influence of serotype on the growth kinetics and the ability to form biofilms of Salmonella 
isolates from poultry. Food Microbiol 2012; 31 : 173-80.

39.- Zou Y, Woo J, Ahn J. Cellular and molecular responses of Salmonella Typhimurium to antimicrobial-induced stresses during the planktonic-to-biofilm transition. Lett Appl Microbiol. 2012; 55: 274-82.

40.- Rodrigues D, Cerca N, Teixeira P, Oliveira R, Ceri H, Azeredo J. Listeria monocytogenes and Salmonella enterica Enteritidis biofilms susceptibility to different disinfectants and stress-response and virulence gene expression of surviving cells. Microb Drug Resist 2011; 17: 181-9.

41.- Aviles B, Klotz C, Eifert J, Williams R, Ponder M. Biofilms promote survival and virulence of Salmonella enterica sv. Tennessee during prolonged dry storage and after passage through an in vitro digestion system. Int J Food Microbiol 2013; 162: 252-9.

42.- Roberfroid S, Hermans K, Robijns S C, Steenackers H P, Vanderleyden J, De Keersmaecker S C. Towards understanding gene expression in multispecies biofilms containing Salmonella Typhimurium. Commun Agric Appl Biol Sci 2012; 77: 33-7.

43.- WHO. Critically important antimicrobials for human medicine 2012. Disponible en: http://apps.who.int/iris/ bitstream/10665/77376/1/9789241504485_eng. pdf?ua $=1 \& u a=1$.

44.- CDC. Antibiotic Resistance Threats in the United States, 2013. Disponible en: http:// www.cdc.gov/drugresistance/threat-report-2013/ index.html2014 [cited 2015 Dec 1st]

45.- Fresno M, Barrera V, Gornall V, Lillo P, Paredes N, Abalos P, et al. Identification of diverse Salmonella serotypes, virulotypes, and antimicrobial resistance phenotypes in waterfowl from Chile. Vector Borne Zoonotic Dis 2013; 13: 884-7.

46.- Dougnac C, Pardo C, Meza K, Arredondo C, Blank O, Abalos P, et al. Detection of Salmonella enterica in Magellanic penguins (Spheniscus magellanicus) of Chilean Patagonia: evidences of inter-species transmission. Epidemiol Infect 2015; 143: 1187-93.

47.- Retamal P, Fresno M, Dougnac C, Gutiérrez S, Gornall V, Vidal R, et al. Genetic and phenotypic evidence of the Salmonella enterica serotype Enteritidis human-animal interface in Chile. Front Microbiol 2015; 6.

48.- Tello A, Austin B, Telfer T C. Selective pressure of antibiotic pollution on bacteria of importance to public health. Environ Health Persp 2012; 120: 1100-6.

49.- Andersson D I, Hughes D. Microbiological effects of sublethal levels of antibiotics. Nature Rev Microbiol 2014; 12: 465-78.

50.- Rodríguez I, Rodicio M R, Guerra B, Hopkins K L. Potential international spread of multidrug- resistant invasive Salmonella enterica serovar Enteritidis. Emerg Infect Dis 2012; 18: 1173-6.

51.- Gullberg E, Albrecht L M, Karlsson C, Sandegren L, Andersson D I. Selection of a multidrug resistance plasmid by sublethal levels of antibiotics and heavy metals. mBio. 2014; 5: e01918-01914.

52.- Wellington E M, Boxall A B, Cross P, Feil E J, Gaze W H, Hawkey P M, et al. The role of the natural environment in the emergence of antibiotic resistance in gram-negative bacteria. Lancet Infect Dis 2013; 13: 155-65.

53.- Borie C, Sánchez M L, Navarro C, Ramírez S, Morales M A, Retamales J, et al. Aerosol spray treatment with bacteriophages and competitive exclusion reduces Salmonella Enteritidis infection in chickens. Avian Dis 2009; 53 : 250-4.

54.- Wells J E, Berry E D, Guerini M N, Varel V H. Evaluation of essential oils in beef cattle manure slurries and applications of select compounds to beef feedlot surfaces to control zoonotic pathogens. J Appl Microbiol 2015; 118: 295 304.

55.- Jones B A, Grace D, Kock R, Alonso S, Rushton J, Said M Y, et al. Zoonosis emergence linked to agricultural intensification and environmental change. Proc Natl Acad Sci U S A. 2013; 110: 8399-404.

56.- Horton R A, Wu G, Speed K, Kidd S, Davies $\mathrm{R}$, Coldham N G, et al. Wild birds carry similar Salmonella enterica serovar Typhimurium strains to those found in domestic animals and livestock. Res Vet Sci 2013; 95: 45-8.

57.- Farías L F, Oliveira C J, Medardus J J, Molla B Z, Wolfe B A, Gebreyes W A. Phenotypic and genotypic characterization of Salmonella enterica in captive wildlife and exotic animal species in Ohio, USA. Zoon Public Health 2015; 62: 438-44.

58.- Fuller T, Bensch S, Müller I, Novembre J, Pérez-Tris J, Ricklefs R E, et al. The ecology of emerging infectious diseases in migratory birds: an assessment of the role of climate change and priorities for future research. Ecohealth 2012; 9: 80-8.

59.- Hubálek Z. An annotated checklist of pathogenic microorganisms associated with migratory birds. J Wildl Dis 2004; 40: 639-59.

60.- Kobayashi H, Kanazaki M, Shimizu Y, Nakajima H, Khatun M M, Hata E, et al. Salmonella isolates from cloacal swabs and footpads of wild birds in the immediate environment of Tokyo Bay. J Vet Med Sci 2007; 69: 309-11.

61.- Pennycott T W, Park A, Mather H A. Isolation of different serovars of Salmonella enterica from wild birds in Great Britain between 1995 and 2003. Vet Rec 2006; 158: 817-20.

62.- Clark R G, Swanney S, Nicol C M, Leyland M, Fenwick S G. Salmonella Brandenburgemergence of a variant strain on a sheep farm in the South Island of New Zealand. N Z Vet J 2003; 51: 146-7.

63.- Millán J, Aduriz G, Moreno B, Juste R A, Barral M. Salmonella isolates from wild birds and mammals in the Basque Country (Spain). Rev Sci Tech 2004; 23: 905-11.

64.- Reche M P, Jiménez P A, Álvarez F, García de los Ríos J E, Rojas A M, de Pedro P. Incidence of Salmonellae in captive and wild free-living raptorial birds in central Spain. J Vet Med B Infect Dis Vet Public Health 2003; 50: 42-4.

65.- Hall A J, Saito E K. Avian wildlife mortality events due to salmonellosis in the United States, 1985-2004. J Wildl Dis 2008; 44: 585-93.

66.- Hughes L A, Shopland S, Wigley P, Bradon $\mathrm{H}$, Leatherbarrow A H, Williams N J, et al. Characterisation of Salmonella enterica serotype Typhimurium isolates from wild birds in northern England from 2005-2006. BMC Vet Res 2008; 4: 4.

67.- Reed K D, Meece J K, Henkel J S, Shukla S K. Birds, migration and emerging zoonoses: West Nile virus, Lyme disease, influenza A and enteropathogens. Clin Med Res 2003; 1 : 5-12.

68.- van Andel M, Jackson B H, Midwinter A C, Alley M R, Ewen J G, McInnes K, et al. Investigation of mortalities associated with Salmonella spp. infection in wildlife on Tiritiri Matangi Island in the Hauraki Gulf of New Zealand. N Z Vet J 2015; 63: 235-9.

69.- López-Martín J, Junod T, Riquelme F, Contreras C, González-Acuña D. Detección de especies de Salmonella y Mycobacterium en gaviotas dominicanas (Larus dominicanus) y gaviotas de Franklin (Leucophaeus pipixcan) en la ciudad de Talcahuano, Chile. Rev Med Chile 2011; 139: 1496-502.

70.- Rodríguez F, Moreno J, Ortega R, Mathieu C, García A, Cerda-Leal F, et al. Evidence for Kelp Gulls (Larus dominicanus) and Franklin's Gulls (Leucophaeus pipixcan) as carriers of Salmonella by real-time polymerase chain reaction. J Wildl Dis 2012; 48: 1105-8.

71.- Fresno M, Barreto M, Gutiérrez S, Dougnac C, Abalos P, Retamal P. Serotype-associated polymorphisms in a partial rpoB gene sequence of Salmonella enterica. Can J Microbiol 2014; 60: 177-81.

72.- MINSAL. Boletín Epidemiológico Trimestral. 2016. Disponible en http://epi.minsal.cl/wpcontent/uploads/2016/05/BET-ETA-ENERO MARZO-2016.pdf

73.- Alerte V, Cortés A S, Díaz T J, Vollaire Z J, Espinoza M E, Solari G V, et al. Brotes de enfermedades transmitidas por alimentos y agua en la Región Metropolitana, Chile (2005-2010). Rev Chilena Infectol 2012; 29: 26-31.

74.- Sturm N, Abalos P, Fernández A, Rodríguez G, Oviedo P, Arroyo V, et al. Salmonella enterica in Pinnipeds, Chile. Emerg Infect Dis 2011; 17: 2377-8. 
75.- Tompkins D M, Carver S, Jones M E, Krkošek M, Skerratt L F. Emerging infectious diseases of wildlife: a critical perspective. Trends Parasitol 2015; 31: 149-59.

76.- Li B, Vellidis G, Liu H, Jay-Russell M, Zhao S, Hu Z, et al. Diversity and antimicrobial resistance of Salmonella enterica isolates from surface water in Southeastern United States. Appl Environ Microbiol 2014; 80: 6355-65.

77.- Jokinen C C, Koot J, Cole L, Desruisseau A, Edge T A, Khan I U, et al. The distribution of Salmonella enterica serovars and subtypes in surface water from five agricultural regions across Canada. Water Res 2015; 76: 120-31.

78.- Williams M S, Ebel E D. Estimating the correlation between concentrations of two species of bacteria with censored microbial testing data. Int J Food Microbiol 2014; 175 : 1-5.

79.- Pachepsky Y, Shelton D, McLain J, Patel J, Mandrell R. Irrigation waters as a source of pathogenic microorganisms in produce: a review. Adv Agronomy 2011; 113: 73-138.

80.- Fica A, Acosta G, Dabanch J, Perret C, Torres M, López J, et al. Brotes de salmonelosis y el tamaño y rol del Estado en Chile. Rev Chilena Infectol 2012; 29: 207-14.

81.- Berngruber T W, Froissart R, Choisy M, Gandon S. Evolution of virulence in emerging epidemics. PLoS Pathog. 2013; 9:e1003209.

82.- Sivapalasingam S, Barrett E, Kimura A, Van Duyne S, De Witt W, Ying M, et al. A multistate outbreak of Salmonella enterica serotype Newport infection linked to mango consumption: impact of water-dip disinfestation technology. Clin Infect Dis 2003; 37: 1585-90.
83.- Hodges J R, Kimball A M. The global diet: trade and novel infections. Global Health 2005; 1: 4.

84.- Chomel B B. Emerging and re-emerging zoonoses of dogs and cats. animals 2014; 4 : 434-45.

85.- Procter T D, Pearl D L, Finley R L, Leonard E $\mathrm{K}$, Janecko N, Reid-Smith R J, et al. A crosssectional study examining Campylobacter and other zoonotic enteric pathogens in dogs that frequent dog parks in three cities in southwestern Ontario and risk factors for shedding of Campylobacter spp. Zoon Public Health 2014; 61: 208-18.

86.- Paris J K, Wills S, Balzer H J, Shaw D J, GunnMoore D A. Enteropathogen co-infection in UK cats with diarrhoea. BMC Vet Res 2014; 10: 13.

87.- Subsecretaría de Desarrollo Regional y Administrativo, Gobierno de Chile. 2014. Disponible en http://www.munitel.cl/noticias/ Tenenciaresponsableanimales.pdf.

88.- Nemser S M, Doran T, Grabenstein M, McConnell T, McGrath T, Pamboukian R, et al. Investigation of Listeria, Salmonella, and toxigenic Escherichia coli in various pet foods. Foodborne Pathog Dis 2014; 11: 706-9.

89.- Hamilton-West C, Rojas H, Pinto J, Orozco J, Hervé-Claude L P, Urcelay S. Characterization of backyard poultry production systems and disease risk in the central zone of Chile. Res Vet Sci 2012; 93: 121-4.

90.- Chomel B B, Belotto A, Meslin F X. Wildlife, exotic pets, and emerging zoonoses. Emerg Infect Dis 2007; 13: 6-11.

91.- CDC. Eight multistate outbreaks of human Salmonella infections linked to small turtles
(Final Update). Disponible en: http://www. cdc.gov/Salmonella/small-turtles-03-12/index. html2013 [cited 201525 Nov 2015]

92.- Osorio M, Saldías M, Valiente K. Estudio retrospectivo de presencia de Salmonella spp. en reptiles ingresados a Chile a través de la Estación Cuarentenaria Pecuaria, 1997-2008. Boletín Veterinario Oficial 2010; 12: 1-10.

93.- Braun S, Spalloni W, Ferreccio F, Postigo J, Fernández A, Porte L, et al. Gastroenteritis por Salmonella spp. en tres lactantes asociada a contacto con tortugas acuáticas. Rev Chilena Infectol 2015; 32: 334-8.

94.- CDC. Multistate outbreak of Salmonella Paratyphi B variant $\mathrm{L}(+)$ tartrate $(+)$ and Salmonella Weltevreden infections linked to frozen raw tuna (Final Update). Disponible en http://www.cdc.gov/Salmonella/ paratyphi-b-05-15/index.html2015 [cited 2015 25 Nov 2015].

95.- García-Huidobro D, Carreño M, Alcayaga S, Ulloa J. Descripción clínica y epidemiológica de un grave brote de salmonelosis transmitida por alimentos. Rev Chilena Infectol 2012; 29: 132-7.

96.- EFSA. Salmonella. Disponible en http://www. efsa.europa.eu/en/topics/topic/Salmonella2011.

97.- Ministerio de Salud. Estrategia nacional de salud para el cumplimiento de los objetivos sanitarios de la década 2011-2020. Disponible en http://web.minsal.cl/portal/url/item/ c4034eddbc96ca6de0400101640159b8.pdf

98.- Villalobos M P, Rojas M A, Leporati N M. Chile potencia agroalimentaria: compromiso con la nutrición y la salud de la población. Rev Chilena Nutr 2006; 33: 232-7. 